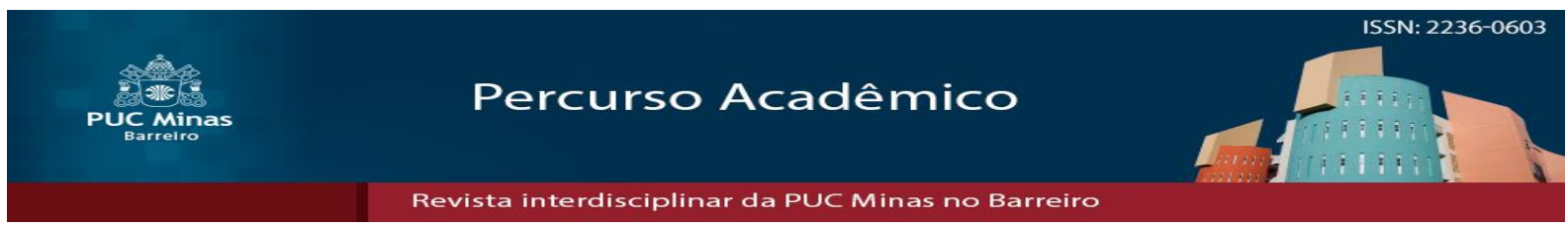

\title{
O estado atual da cirurgia robótica colorretal
}

\section{The present state of colorectal robotic surgery}

\author{
Larissa Gonçalves Rezende ${ }^{1}$ \\ Eduardo de Aquino Médici ${ }^{1}$ \\ Beatriz Deoti e Silva Rodrigues ${ }^{2}$
}

\begin{abstract}
RESUMO
INTRODUÇÃO: Nas últimas décadas, houve um crescimento da cirurgia minimamente invasiva e, mais recentemente, da cirurgia laparoscópica assistida por robótica. Mesmo com esses avanços alcançando diversas áreas, a maioria das cirurgias colorretais ainda são feitas por via aberta decorrente dos desafios técnicos e das incertezas sobre os desfechos das novas técnicas no resultado da cirurgia do câncer retal. DESENVOLVIMENTO: Analisar viabilidade, segurança e eficácia da robótica, com ênfase às suas aplicações na cirurgia colorretal por meio dos resultados publicados das variáveis taxas de complicações; de conversão; de reoperação; de mortalidade; de desfechos funcionais nas esferas urinária, sexual e fecal e oncológicas de sobrevida global ou livre da doença. METODOLOGIA: Revisão de literatura nas bases de dados eletrônicas PubMed e Cochrane. Os descritores foram "robotic", "robotic versus laparoscopic" AND "colorectal surgery" e a seleção dos estudos publicados foi a partir do ano de 2015. RESULTADOS: Não houve diferença estatisticamente significante quando comparados os grupos da cirurgia robótica e laparoscópica nos quesitos de complicações e desfechos oncológicos. Não houve diferença significativa entre os grupos quando comparadas as complicações peri e pós-operatórias dos dois grupos. DISCUSSÃO: Na cirurgia robótica do câncer de reto, movimentos como o punho permitem destreza no manuseio da pelve. Embora permita recuperação, retorno à função intestinal normal e retomada da alimentação mais rápidos, os desfechos oncológicos são semelhantes pelas vias robótica, laparoscópica e aberta. CONCLUSÃO: Os resultados obtidos pela cirurgia robótica são positivos, no mínimo equivalentes às técnicas já estabelecidas. Por ser uma nova tecnologia, torna-se necessário validar por meio de estudos de alta qualidade, clínicos randomizados controlados, os
\end{abstract}

Artigo recebido em 01 de outubro de 2019 e aprovado em 16 de dezembro de 2019

${ }^{1}$ Acadêmicos da Faculdade de Medicina da Universidade Federal de Minas Gerais.

${ }^{2}$ Professora Adjunta do Departamento de Cirurgia da Faculdade de Medicina da Universidade Federal de Minas Gerais. 
desfechos da cirurgia robótica colorretal e acompanhar a evolução e as novidades da robótica que estão por vir.

Palavras-chave: Robótica. Robótica versus laparoscópica. Cirurgia colorretal.

\begin{abstract}
INTRODUCTION: In the last decades, there has been a growth in minimally invasive surgery and, more recently, robot assisted surgery. Even with these advances reaching several areas, most colorectal surgeries are still performed openly due to technical challenges and uncertainties about the outcomes of new techniques without rectal surgery results. DEVELOPMENT: Analyze the feasibility, safety and efficacy of robotics, with emphasis on its applications in colorectal surgery through published results of complications; of conversion; reoperation; of mortality; of functional outcomes in the urinary, sexual and fecal and oncological spheres of global or disease-free survival. METHODOLOGY: Literature review in PubMed and Cochrane electronic databases. The descriptors were "robotic", "robotic versus laparoscopic" AND "colorectal surgery" and the published studies were selected from 2015. RESULTS: There was no statistically significant difference when comparing the robotic and laparoscopic surgery groups considering complications and cancer outcomes. There was no significant difference between groups when compared as peri and postoperative complications of two groups. DISCUSSION: In robotic surgery of rectal cancer, movements such as the wrist may affect the handling of the pelvis. While allowing recovery, return to normal bowel function, and recovery from faster eating, there is no difference between cancer outcomes in robotic, laparoscopic, and open pathways. CONCLUSION: The results obtained by robotic surgery are positive, at least equivalent to what it does. Because it is a new technology, it is necessary to assess through high-quality, prospective randomized controlled trials, the outcomes of colorectal robotic surgery and to monitor the evolution and news of forthcoming robotics.
\end{abstract}

Key-words: Robotic. Robotic versus laparoscopic. Colorectal surgery.

\title{
1 INTRODUÇÃO
}

A palavra robótica é de origem tcheca e significa "trabalho forçado" e foi usada pela primeira vez na história pelo tcheco Karel apeCapek, numa peça de teatro em 1921, em Praga, data esta não totalmente precisa. Em 1495, Leonardo da Vinci fez um projeto de um autônomo humanóide e, atualmente, o aparelho novo leva o seu nome. Outros construtores que já haviam 
apresentado seus humanóides séculos antes, mais notavelmente, foram Albertus Magnus, Roger Bacon e Villard d'Honnecourt (CHENG, 2018). Nas últimas décadas, a cirurgia robótica vem sendo considerada uma nova modalidade para superar as limitações técnicas da cirurgia convencional. Apesar desse novo arsenal, a maioria das cirurgias colorretais ainda são feitas por via aberta, decorrente dos desafios técnicos para realizar esse tipo de acesso cirúrgico e ainda da falta de evidências sobre os desfechos das novas técnicas na cirurgia do câncer colorretal. A análise da viabilidade, da eficácia e da segurança desse acesso cirúrgico é oportuna para se entender o estado atual da robótica no tratamento da doença colorretal (MORAN, 2007) (CHENG, 2018) (PARK, 2015) (JAYNE, 2017).

\section{DESENVOLVIMENTO}

Diante das inovações tecnológicas da cirurgia robótica, tornam-se necessários constantes estudos para validar ou rejeitar algumas de suas aplicações. O objetivo foi analisar os resultados publicados das variáveis taxas de complicações; de conversão; de reoperação; de mortalidade; de desfechos funcionais nas esferas urinária, sexual e fecal e oncológicas de sobrevida global ou livre da doença com o objetivo de avaliar viabilidade, segurança e eficácia da cirurgia robótica colorretal.

\section{METODOLOGIA}

Revisão de literatura nas bases de dados eletrônicos PubMed e Cochrane, utilizando os descritores "robotic", "robotic versus laparoscopic" AND "colorectal surgery". A seleção dos artigos publicados foi a partir de 2015.

As variáveis avaliadas foram as taxas de complicações intraoperatórias, complicações até 30 dias da cirurgia, complicações entre 30 dias e 6 meses do procedimento, conversões, reoperações e mortalidade. A variável desfechos oncológicos refere-se ao número de linfonodos recuperados, margens positivas e readmissão ou mortalidade em 30 dias. Foram avaliados os desfechos sexuais por meio do International Index Erectile Function (IIEF) em homens e 
do Female Sexual Function Index (FSFI) em mulheres e os desfechos urinários em homens por meio do International Prostate Symptom Score (I-PSS).

\section{RESULTADOS}

Os resultados obtidos foram agrupados na tabela 1, comparando os desfechos pela via laparoscópica convencional e laparoscópica assistida por robótica.

Tabela 1: Resultados das complicações listadas nos artigos analisados

\begin{tabular}{|c|c|c|c|c|c|}
\hline Autores & $\begin{array}{l}\text { PARK, Eun } \\
\text { et al }\end{array}$ & $\begin{array}{l}\text { ZHANG, } \\
\text { Xuan et al }\end{array}$ & $\begin{array}{c}\text { ASKLID, } \\
\text { Daniel et al }\end{array}$ & $\begin{array}{l}\text { JAYNE, } \\
\text { David et al }\end{array}$ & $\begin{array}{c}\text { AHMED, J } \\
\text { et al }\end{array}$ \\
\hline Ano & 2014 & 2016 & 2017 & 2017 & 2017 \\
\hline Complicações & $\begin{array}{l}\text { Perioperató } \\
\text { rias e pós } \\
\text { operatórias } \\
\text { a longo } \\
\text { prazo }\end{array}$ & $\begin{array}{l}\text { Intraoperatór } \\
\text { iase pós- } \\
\text { operatórias }\end{array}$ & $\begin{array}{c}\text { A curto } \\
\text { prazo }\end{array}$ & $\begin{array}{c}\text { Intraopera } \\
\text { tórias, até } \\
30 \text { dias de } \\
\text { pós- } \\
\text { operatório } \\
\text { e } 30 \text { dias } \\
\text { a } 6 \text { meses } \\
\text { de pós- } \\
\text { operatório }\end{array}$ & $\begin{array}{c}\text { Pós- } \\
\text { operatórias } \\
\text { em } \\
\text { pacientes } \\
\text { de alto risco }\end{array}$ \\
\hline Pacientes & $\begin{array}{c}\mathrm{n}=133 \\
\text { (robótica) } \\
\mathrm{n}=84 \\
\text { (laparoscóp } \\
\text { ica) }\end{array}$ & $\begin{array}{c}\mathrm{n}=1466 \\
\text { (robótica) } \\
\mathrm{n}=1852 \\
\text { (laparoscópi } \\
\text { ca) }\end{array}$ & $\begin{array}{c}\mathrm{n}=72 \\
\text { (robótica) } \\
\mathrm{n}=47 \\
\text { (laparoscó } \\
\text { pica) }\end{array}$ & $\begin{array}{c}\mathrm{n}=236 \\
\text { (robótica) } \\
\mathrm{n}=230 \\
\text { (laparoscó } \\
\text { pica) }\end{array}$ & $\begin{array}{c}\mathrm{n}=47 \\
\text { (laparoscóp } \\
\text { ica) } \\
\mathrm{n}=72 \\
\text { (robótica) }\end{array}$ \\
\hline Conclusão & $\begin{array}{l}\text { Não houve } \\
\text { diferença } \\
\text { significativa } \\
\text { entre os } \\
\text { grupos }\end{array}$ & $\begin{array}{l}\text { Não houve } \\
\text { diferença } \\
\text { significativa } \\
\text { entre os } \\
\text { grupos }\end{array}$ & $\begin{array}{c}\text { Menos } \\
\text { complicaçõ } \\
\text { es na } \\
\text { cirurgia } \\
\text { robótica }\end{array}$ & $\begin{array}{c}\text { Não } \\
\text { houve } \\
\text { diferença } \\
\text { significativ } \\
\text { a entre os } \\
\text { grupos }\end{array}$ & $\begin{array}{c}\text { Menos } \\
\text { complicaçõ } \\
\text { es na } \\
\text { cirurgia } \\
\text { robótica }\end{array}$ \\
\hline
\end{tabular}

Fonte: Elaborado pela autora 
Nas variáveis taxas de complicações intraoperatórias, complicações até 30 dias da cirurgia, complicações entre 30 dias e 6 meses do procedimento, conversões, reoperações e mortalidade, assim como nos desfechos oncológicos, os achados estatísticos tiveram níveis descritivos acima de 0.05, tendo baixa relevância estatística (Tabela 2). Também não houve diferença estatisticamente significante entre os grupos quando foram avaliados os desfechos sexuais e urinários.

Tabela 2: Montada com base nos resultados de PARK, 2014 e JAYNE, 2017

$\begin{array}{lll}\text { Variável } & \begin{array}{l}\text { Laparoscópica } \\ \text { convencional }\end{array} & \begin{array}{l}\text { Laparoscópica } \\ \text { assistida por p-valor } \\ \text { robótica }\end{array}\end{array}$

\begin{tabular}{lccc}
\hline $\begin{array}{l}\text { Complicações } \\
\text { intraoperatórias }\end{array}$ & $14,8 \%^{4}$ & $15,3 \%^{4}$ & 0,944 \\
$\begin{array}{l}\text { Complicações até } \\
30 \text { dias da cirurgia }\end{array}$ & $31,7 \%^{4}$ & $33,1 \%^{4}$ & 0,844 \\
$\begin{array}{l}\text { Complicações } \\
\text { entre 30 dias e } 6\end{array}$ & $16,5 \%{ }^{4}$ & $14,4 \%^{4}$ & 0,254 \\
meses de cirurgia & & & \\
Conversões & $12,2 \%{ }^{4}$ & $8,1 \%{ }^{4}$ & 1,604 \\
Reoperações & $4,7 \%{ }^{3}$ & $4 \%{ }^{3}$ & 1,03 \\
Mortalidade & $0,9 \%^{4}$ & $0,9 \%^{4}$ & - \\
Desfechos & $6,2 \%{ }^{4}$ & $5,1 \%{ }^{4}$ & 0,564 \\
oncológicos & & & \\
\hline
\end{tabular}

Fonte: Elaborado pela autora 
De acordo com a Escala de Clavien-Dindo, as complicações são classificadas em graus: I (qualquer desvio do curso pós-operatório ideal que não necessite de tratamento farmacológico ou de intervenções cirúrgicas, endoscópicas ou radiológicas; terapias aceitas aqui são: drogas antieméticas, antipiréticos, analgésicos, diuréticos, eletrólitos e fisioterapia), II (requer terapia farmacológica diferente da permitida para a categoria I, transfusão sanguínea ou nutrição parenteral), III (exige intervenção cirúrgica, endoscópica ou radiológica), IV (complicação com risco de vida) e V (morte do paciente). No estudo de PARK, 2014, foram listadas complicações grau I, como dificuldade de urinar, infecção de ferida, derrame pleural, ejaculação retrógrada e obstrução intestinal; grau II, ascite e hemotransfusão; e grau III, deiscência de anastomose, hemorragia consequente, colite isquêmica, abscesso intraabdominal, aderência e hérnia incisional.

Em apenas um dos estudos (PARK, 2014), houve uma análise da incidência de cada tipo de complicação pós-operatória em específico, independente do seu tempo de ocorrência. Tal fato viabilizou a comparação desses dados considerando o grau da complicação por meio da escala de Clavien-Dindo (MOREIRA, 2016).

\section{DISCUSSÃo}

A cirurgia robótica apresenta vantagens, como a liberdade de movimentos (semelhante ao punho), ergonomia superior, visão tridimensional, controle do campo operatório, maior autonomia do cirurgião e maior precisão com movimentos fluidos e acurados devido à interface computacional. Entretanto, algumas desvantagens da técnica são os custos elevados para compra e manutenção dos aparelhos; tempo cirúrgico prolongado; curva de aprendizado longa, cujo processo envolve treinamento em simuladores, assistência na cabeceira e prática no console duplo com mentor até que o cirurgião esteja habilitado a realizar os procedimentos sozinho; ausência de feedback tátil, podendo ocorrer a lesão de tecidos; e falta de guidelines com uma padronização do método e critérios para sua reprodutibilidade (CHENG, 2018) (PAI, 2017) (ZAWADZKI, 2017) (ZHANG, 2016). 
Em pacientes de alto risco como portadores de câncer de reto baixo localmente avançado (T3/T4), após tratamento neoadjuvante, com IMC maior do que $30 \mathrm{~kg} / \mathrm{m}^{2}$, com cirurgia abdominal prévia, do sexo masculino, a literatura mostra que a abordagem robótica pode ter benefícios adicionais (CHENG, 2018) (ASKILD, 2018) (MOREIRA, 2016).

O ensaio clínico randomizado do câncer retal não demonstrou nenhum benefício para a ressecção retal robótica em comparação com a ressecção laparoscópica convencional em mais de uma variável - número de linfonodos recuperados, margens positivas e readmissão ou mortalidade em 30 dias. Existem expectativas sobre melhores resultados na ressecção circunferencial das margens, associada aos índices de recorrência e sobrevida a longo prazo, quando realizada por robôs; porém, ainda são necessários mais estudos para confirmação (CHENG, 2018) (PARK, 2014) (JAYNE, 2017) (ZHANG, 2016) (DE JESUS, 2016).

Deve-se conhecer a taxa de complicações e reoperações para validar uma abordagem cirúrgica específica. Para isso, os dados sobre complicações precisam ser analisados de acordo com sua gravidade e considerando os tipos de complicações em específico. Apenas um dos artigos utilizou dessa abordagem por meio da Escala de Clavien-Dindo. Além disso, é necessária uma definição clara dos tipos de complicações intraoperatórias e sua gravidade. É importante que os estudos continuem para esclarecer o impacto da experiência dos cirurgiões envolvidos nos desfechos da cirurgia robótica. Este é um dos grandes desafios, devido à dificuldade de padronizar o estudo e de alcançar resultados que possam ser comparados. Para um mesmo cirurgião, por exemplo, podem haver diferenças de habilidade e destreza entre a cirurgia aberta, laparoscópica e robótica, sendo ele melhor ou pior em alguma delas. Do mesmo modo, quando são comparadas as três vias de acesso com cada uma sendo executada por cirurgiões diferentes, esse viés também permanece (PARK,2014) (JAYNE, 2017).

Os resultados devem partir de um número homogêneo de procedimentos realizados por centro e é preciso ter uma média maior do número de cirurgias por centro. $O$ baixo número de procedimentos não pode ser considerado suficiente para tirar conclusões sobre a eficácia da abordagem 
robótica, haja vista que um centro de alto volume realiza pelo menos 20 a 30 procedimentos por ano.

É fundamental que a experiência dos cirurgiões e a padronização da técnica operatória e do cuidado perioperatório devam ser os primeiros fatores a serem considerados no planejamento dos próximos estudos, pois, assim, poderá ser possível avaliar se a cirurgia robótica de câncer retal se justifica por mais que apresente altos custos. (ZHANG, 2016) (JAYNE, 2017)

Os resultados existentes demonstram que, no mínimo, a cirurgia robótica é igual as padronizadas, isso porque está na fase embrionária. Nas últimas duas décadas, a cirurgia robótica promoveu uma revolução notável na maneira de executar, ensinar e praticar cirurgia. Apesar de algumas limitações, como por exemplo a ausência de sensação tátil, a proposta robótica é um método de tratamento comprovado. Isso é só o começo de muita novidade que está por surgir, como o uso da inteligência artificial, um robô capaz de localizar uma lesão de órgão sem ajuda humana, o uso de nanorrobôs, que são robôs do tamanho de uma célula que podem ser introduzidos na corrente sanguínea para eliminar células cancerígenas, reparar tecidos ou capturar radicais tóxicos, todos guiados por controle remoto (MORAN, 2007).

Em constante desenvolvimento por várias décadas, a cirurgia robótica faz parte de uma evolução natural e lógica da cirurgia minimamente invasiva, fornecendo soluções para suas limitações e, assim, melhorando a destreza do cirurgião e a precisão do procedimento cirúrgico. Em um futuro próximo, contribuirá para o progresso técnico no desenvolvimento e propagação de cirurgias de incisão única e até sem cicatrizes (CHENG, 2018).

A interposição de uma interface eletromecânica entre paciente e cirurgião, por meio da cirurgia robótica, constitui uma grande mudança e já provou que é capaz de acrescentar e evoluir.

\section{CONSIDERAÇÕES FINAIS}

No que diz respeito à cirurgia robótica, ainda há muito a ser incorporado na técnica, investigado e desenvolvido, mas os resultados atuais obtidos são positivos, no mínimo, equivalente ao que se faz. Parece ser apenas uma 
questão de tempo até que a cirurgia robótica se torne o procedimento de referência para um número significativo de intervenções cirúrgicas. Por ser uma nova tecnologia, torna-se necessário evoluir nos estudos e avaliar por meio de pesquisas de alta qualidade, prospectivos randomizados controlados, os desfechos da cirurgia robótica colorretal.

\section{REFERÊNCIAS}

MORAN, Michael E. The da Vinci Robot. Journal of Endourology: Epochs in Endourology, [s. I.], v. 20, n. 12, 5 jan. 2007. Disponível em:

https://doi.org/10.1089/end.2006.20.986 Acesso em: 28 set. 2019.

CHENG, Christina L; REZAC, Craig. The role of robotics in colorectal surgery. BMJ, 12 fev. 2018. DOI 10.1136/bmj.j5304. Disponível em: https://www.bmj.com/content/360/bmj.j5304 Acesso em: 27 set. 2019.

PARK, Eun et al. Long-term Oncologic Outcomes of Robotic Low Anterior Resection for Rectal Cancer: A Comparative Study With Laparoscopic Surgery. Annals of surgery, [s. I.], v. 261, n. 1, p. 129-137, 1 jan. 2015. DOI 10.1097/SLA.0000000000000613. Disponível em:

https://insights.ovid.com/pubmed?pmid=24662411 Acesso em: 28 set. 2019.

JAYNE, David et al. Effect of Robotic-Assisted vs Conventional Laparoscopic Surgery on Risk of Conversion to Open Laparotomy Among Patients Undergoing Resection for Rectal Cancer: The ROLARR

Randomized Clinical Trial. JAMA, [s. I.], v. 318, n. 16, p. 1569-1580, 24 out. 2017. DOI 10.1001/jama.2017.7219. Disponível em:

https://jamanetwork.com/journals/jama/fullarticle/2658320 Acesso em: 28 set. 2019.

PAl, Ajit et al. Robotic Surgery for Colon and Rectal Cancer: Current Status, Recent Advances, and Future Directions. Current Colorectal Cancer Reports, Nova lorque, v. 13, n. 1, p. 37-44, 4 fev. 2017. DOI 10.1007/s11888-017-0348- 
7. Disponível em: https://www.springermedizin.de/robotic-surgery-for-colon-andrectal-cancer-current-status-recen/12044894 Acesso em: 27 set. 2019.

ZAWADZKI, Marek et al. Comparison of inflammatory responses following robotic and open colorectal surgery: a prospective study. International Journal of Colorectal Disease, [s. I.], v. 32, n. 3, p. 399-407, 4 nov. 2017. DOI 10.1007/s00384-016-2697-0. Disponível em:

https://link.springer.com/article/10.1007/s00384-016-2697-0 Acesso em: 27 set. 2019.

ZHANG, Xuan et al. Robot-assisted versus laparoscopic-assisted surgery for colorectal cancer: a meta-analysis. Surgical Endoscopy, [s. I.], v. 30, n. 12, p. 5601-5614, 1 dez. 2016. DOI 10.1007/s00464-016-4892-z. Disponível em: https://link.springer.com/article/10.1007/s00464-016-4892-z Acesso em: 27 set. 2019.

ASKILD, Daniel et al. Robotic vs laparoscopic rectal tumour surgery: a cohort study. Colorectal disease, [s. I.], v. 21, n. 2, p. 191-199, 14 nov. 2018. DOI 10.1111/codi.14475. Disponível em: https://onlinelibrary.wiley.com/doi/abs/10.1111/codi.14475 . Acesso em: 27 set. 2019.

AHMED, J et al. Robotic vs laparoscopic rectal surgery in high: risk patients. Colorectal disease, [s. I.], v. 19, n. 12, p. 1092-1099, 23 jun. 2017. DOI 10.1111/codi.13783. Disponível em:

https://onlinelibrary.wiley.com/doi/abs/10.1111/codi.13783 . Acesso em: 28 set. 2019.

MOREIRA, LUIS FERNANDO et al . Cultural adaptation and the ClavienDindo surgical complications classification translated to Brazilian Portuguese. Rev. Col. Bras. Cir., Rio de Janeiro , v. 43, n. 3, p. 141-148, June 2016. Available from http://www.scielo.br/scielo.php?script=sci arttext\&pid=S0100- 
69912016000300141\&lng=en\&nrm=iso access on 28 Sept. 2019. http://dx.doi.org/10.1590/0100-69912016003001

VALERO, R. et al . Cirugía robótica: Historia e impacto en la enseñanza. Actas Urol Esp, v. 35, n. 9, p. 540-545, oct. 2011. Disponible en http://scielo.isciii.es/scielo.php?script=sci arttext\&pid=S021048062011000900006\&lng=es\&nrm=iso accedido en 29 sept. 2019. http://dx.doi.org/10.4321/S0210-48062011000900006.

DE JESUS, J. P. et al. The circumferential resection margins status: A comparison of robotic, laparoscopic and open total mesorectal excision for mid and low rectal cancer. Eur J Surg Oncol., v. 42, n. 6, p. 808-812, jun. 2016. Disponível em https://www.ncbi.nlm.nih.gov/pubmed/27038996 Acesso em 15 dez. 2019. https://doi.org/10.1016/j.ejso.2016.03.002

SÁNCHEZ-MARTIN, F.; VILLAVICENCIO, H. Comments to the article "Robotic surgery: history and impact on teaching". Actas Urol Esp, v. 36, n. 5, p. 331-332, jan. 2012. Disponível em https://www.ncbi.nlm.nih.gov/pubmed/22266255 . Acesso em 29 set. 2019. http://dx.doi.org/10.1016/j.acuro.2011.11.003. 